Itinéraires Itinéraires

Littérature, textes, cultures

\title{
La légende du windigo dans la création autochtone contemporaine au Québec
}

Isabelle St-Amand

\section{(2) OpenEdition}

\section{Journals}

Édition électronique

URL : http://journals.openedition.org/itineraires/2725

DOI : $10.4000 /$ itineraires.2725

ISSN : 2427-920X

Éditeur

Pléiade

Référence électronique

Isabelle St-Amand, "La légende du windigo dans la création autochtone contemporaine au Québec 》, Itinéraires [En ligne], 2015-1 | 2015, mis en ligne le 18 décembre 2015, consulté le 01 mai 2019. URL : http://journals.openedition.org/itineraires/2725; DOI : 10.4000/itineraires.2725

Ce document a été généré automatiquement le 1 mai 2019.

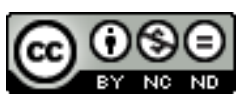

Itinéraires est mis à disposition selon les termes de la licence Creative Commons Attribution - Pas d'Utilisation Commerciale - Pas de Modification 4.0 International. 


\title{
La légende du windigo dans la création autochtone contemporaine au Québec
}

\author{
Isabelle St-Amand
}

La nuit avait fini par tomber. Le dîner avait déjà été servi. Tous avaient pris leur dernière bouchée,

et les aînés passaient le temps. De son côté, le jeune homme ne cessait de parler du windigo qui ne s'était jamais montré le bout du nez. Puis tout à

coup, une peur terrible se fit ressentir. Le jeune homme, surtout, était complètement transi de peur. Il se mis à faire part de sa frayeur à sa mère, mais sa grand-mère répliqua : "Tais-toi ! C'est toi qui l'as voulu!»

Assurément, il y avait un windigo dans les environs. Et, comme c'était habituellement le cas à

l'approche d'un windigo, ils entendirent un battement de cœur - c'était le signe familier qui indiquait, chez nos ancêtres, la proximité d'un windigo. La vieille dame, qui avait reconnu le bruit, déclara: «Tu vois ce que je dis? Tu vois ce que je t'avais dit? C'est toujours ce qui arrive ${ }^{1}$. »

(Bird 2013 : 128)

1 Les pratiques artistiques que sont la littérature et le cinéma autochtones au Québec engagent un domaine de recherche conjuguant éthique de la littérature et pensée créatrice. Dans un contexte marqué par les effets et les répercussions dévastateurs d'un projet colonial incessant, les enjeux d'une création autochtone porteuse à la fois de transformation et de continuité se posent très souvent dans une perspective qui consiste non tant à valoriser l'art pour l'art que de concevoir « l'art pour la vie » (Justice 2004 : 110-111). C'est précisément afin de contrer l'aliénation coloniale, les stéréotypes 
romantiques et la souffrance intergénérationnelle éprouvés par les premiers peuples que l'homme de théatre et réalisateur wendat Yves Sioui Durand en appelle à la force de « l'art comme transmetteur des valeurs éthiques et de nos traditions» (2009:506, 511 et 514). Dans le même esprit, le chercheur ojibwé et dakota Scott Richard Lyons fait valoir l'exercice d'une souveraineté rhétorique capable de guider les peuples autochtones sur "la voie de la liberté d'agir, de la prise de pouvoir et du renouveau communautaire " (Lyons 2000 : 449). Comme le précise Lyons, cette souveraineté ne consiste pas à vouloir ranimer le passé des peuples autochtones, mais bien à faire advenir leurs possibles (Ibid.). Nous proposons ici d'apporter une réflexion issue de la littérature et de la recherche autochtones en Amérique du Nord, puis d'évoquer l'apport d'une telle perspective aux études littéraires.

\section{La littérature autochtone au Québec : une situation de double exiguiité}

2 La littérature autochtone des États-Unis s'est fait connaître en France grâce aux traductions de romans d'écrivains de renom tels Sherman Alexie, N. Scott Momaday et Louise Erdrich. C'est maintenant en traduction tout autant qu'en version originale que des œuvres littéraires autochtones publiées en anglais et en français au nord du $49^{\mathrm{e}}$ parallèle se font de mieux en mieux connaître, ce qui nous incite à examiner ces corpus dans toute leur spécificité. Au Canada, les littératures autochtones d'expression française et anglaise témoignent de la situation politique et culturelle d'un pays construit dans les suites d'une double colonisation, d'abord française, ensuite britannique. Telle qu'exposée dans un dossier de la revue bilingue Études en littérature canadienne, la situation des écrivains autochtones œuvrant dans l'espace francophone du Québec se rapproche et diffère à la fois de la situation de leurs homologues évoluant plutôt dans l'espace anglophone au Canada et au États-Unis :

Tout en partageant avec les peuples autochtones du Canada des conditions historiques, politiques et culturelles, ces auteurs s'inscrivent dans le contexte politique et culturel particulier du Québec, lui-même marqué par son statut minoritaire dans l'ensemble canadien. De ce fait, ils doivent composer avec une double exiguïté : d'une part, les barrières linguistiques issues de la colonisation compliquent les échanges avec le milieu littéraire autochtone d'expression anglaise en Amérique du Nord, d'autre part, l'exiguïté du marché francophone diminue les possibilités de production et de diffusion, ainsi que la masse possible de discours critiques. (St-Amand $2010: 31$ )

3 La réflexion sur les petites cultures et leurs espaces littéraires élaborée par François Paré dans Les Littératures de l'exiguité ([1992] 2001) permet de jeter un éclairage sur la situation particulière des écrivains, cinéastes, artistes, acteurs culturels et politiques et, de façon élargie, des communautés autochtones ayant fait - de gré ou de force - du français leur langue seconde ou première. La dimension autochtone de la création gagne pour sa part à être étudiée en lien avec les productions culturelles, littéraires et intellectuelles autochtones issues de différents espaces linguistiques.

4 En plus de refléter une expérience partagée des politiques et des pratiques coloniales mises en œuvre en Amérique du Nord, la littérature autochtone rend compte de l'existence de peuples précédant et outrepassant les frontières géographiques et culturelles du Québec, du Canada et des États-Unis (St-Amand 2010; DePasquale, Eigenbrod et LaRocque 2009). Ainsi, le recours aux récits et aux langues autochtones dans 
la création autochtone contemporaine, pour ne nommer que cet exemple, rend manifeste et retravaille un fond culturel autre que celui de la tradition occidentale. Tandis que les nombreux travaux réalisés dans l'espace anglophone apportent une force critique indéniable aux études littéraires autochtones au Québec, les liens établis avec des écrivains et des intellectuels de la francophonie autochtone favorisent l'élargissement de l'espace littéraire autochtone d'expression française. En 2008, le Carrefour international des littératures autochtones de la francophonie tenu à Wendake, communauté wendat à proximité de la ville de Québec, a rassemblé des chercheurs de diverses origines, ainsi que des écrivains autochtones du Maroc, de la Polynésie française, de la Nouvelle-Calédonie, de l'Algérie et du Québec, lesquels ont ensuite fait paraître des textes de création dans le recueil Mots de neige, de sable et d'océan (Gatti 2008). Une mise en relation de différentes formes d'art issues des cultures autochtones est également à l'œuvre sur le plan de la création comme de la recherche. Le long métrage Mesnak réalisé par l'homme de théâtre wendat Yves Sioui Durand, l'art-performance du poète wendat Louis-Karl Picard-Sioui et la poésie du réalisateur innu Réal Junior Leblanc insérée dans le court métrage expérimental Chevelure de la vie offrent autant d'exemples de la multiplicité des pratiques. Envisageant la création autochtone dans une perspective multidisciplinaire, le sociologue et commissaire d'art wendat Guy Sioui Durand $(2010: 40)$ met en avant la notion de "peaux visuelles tendues " pour désigner l'artisanat et les beaux-arts, et la notion apparentée de "peaux visuelles animées" pour faire référence à la vidéo, au documentaire, au cinéma et à l'art Web. Ces notions s'arriment à une volonté affirmée de redéfinir les contours de la littérature autochtone de façon à prendre pleinement en compte «la substance imaginante, préhistorique et historique (les pétroglyphes, wampums ${ }^{2}$, codex et les littératures orales transcrites) qui fonde l'imaginaire artistique et, conséquemment, la littérature contemporaine des Indiens d'Amérique » (Sioui Durand 2007: 185). Les récits oraux et les différentes formes d'oralité se prêtent à des remaniements continus que l'on peut voir à l'œuvre dans la littérature et, tout particulièrement, dans le cinéma autochtone francophone en Amérique du Nord.

\section{Constructions coloniales et récits philosophiques}

5 Du point de vue des études autochtones, les notions d'éthique et de souveraineté sont deux notions inter-reliées qui se jouent autant sur le plan politique et social que sur celui de la création et de la recherche. Lyons, en parlant des peuples autochtones, affirme :

La souveraineté est le récit phare qui oriente notre quête d'autodétermination, elle constitue la stratégie d'ensemble par laquelle nous nous efforçons de renverser les pertes causées par les ravages de la colonisation: pertes de nos terres, de nos langues, de nos cultures, de notre respect de soi $^{3}$. (2000:449)

6 Au Canada, les témoignages recueillis auprès des survivants et les faits rendus publics par l'actuelle Commission de vérité et de réconciliation incitent à reconnaître et à remédier à l'ampleur de la dévastation et du trauma intergénérationnel que l'un de ces outils d'assimilation, le système des pensionnats, a causé et continue de causer chez les enfants de même que dans les familles et les communautés des peuples premiers ${ }^{4}$. Dans les excuses présentées par le Canada en 2008, le Premier ministre Stephen Harper reconnaissait que «cette politique a causé des dommages durables à la culture, au patrimoine et à la langue des autochtones ». En plus de faire état de la négligence ainsi que des abus émotionnels, physiques et sexuels infligés à des enfants sans défense, il admettait que les deux principaux objectifs de ces pensionnats, soit l'isolement des 
enfants de leur milieu et leur assimilation à la société dominante, reposaient sur l'hypothèse erronée selon laquelle "les cultures et les croyances spirituelles des Autochtones étaient inférieures ${ }^{5} »$. Les pensionnats autochtones reflétaient en ce sens la mentalité coloniale présidant au projet d'appropriation du territoire et d'effacement des premiers peuples. Eu égard à cette histoire, le chercheur cherokee Daniel Heath Justice fait valoir une lutte autochtone alliant art et politique :

Si notre survie et notre développement nécessitent un vigoureux militantisme économique et politique, ils requièrent aussi un militantisme de l'imagination, un dévouement à nous réaliser hors des mensonges et des stéréotypes coloniaux. (Justice $2004: 109$ )

Précisons que la puissance et l'effectivité de ces stéréotypes sont d'autant plus dommageables qu'ils ne sont pas restreints à la culture populaire, mais qu'ils agissent au cœur même de la pensée politique, des institutions sociétales et de la recherche scientifique.

Dans Revenge of the Windigo: The Construction of the Mind and Mental Health of North American Aboriginal Peoples, l'anthropologue James B. Waldram s'est intéressé à la construction médicale d'une condition appelée la «psychose du windigo». Dans la tradition algonquine, écrit Waldram, le windigo est un monstre cannibale qui rôde dans les forêts, s'empare des gens et les fait disparaître à jamais, la légende voulant que dans certaines circonstances, un être humain puisse se transformer en windigo et dévorer ses proches. Cette légende fait référence, sans s'y limiter, à des situations de famine prolongée pouvant pousser des individus à ingérer de la chair humaine afin d'assurer leur survie. Par un étrange concours de circonstance, explique Waldram, cette légende fut transformée en maladie mentale, la psychose du windigo, laquelle est le plus sérieusement du monde décrite comme un "syndrome lié à la culture» (2004: 17-18). Fait notable, aucun cas clinique de cette prétendue psychose n'a jamais été étudié, de sorte qu'elle a fini par être réfutée (Ibid.: 18). Selon l'anthropologue, la persistance de cette idée illustre parfaitement la façon dont les constructions scientifiques des Autochtones ont fini par s'imposer comme des réalités :

La psychose du windigo est sans doute le meilleur exemple de la construction d'une maladie mentale autochtone par la profession médicale, et sa persistance souligne de façon frappante la façon dont les constructions faites des Autochtones par cette profession ont, comme le monstre de Frankenstein, fini par acquérir une vie qui leur est propre. (Waldram $2004: 18$ )

Si le personnage de Frankenstein évoque l'idée du monstre qui se retourne contre son créateur, la construction médicale inspirée de la légende du windigo ne joue pas contre les scientifiques qui l'ont créée, mais plutôt contre les peuples autochtones dont les récits l'ont inspirée. La psychose du windigo révèle le caractère fictif de certaines constructions médicales, l'ampleur de la méprise et du mépris et, de fait, l'importance fondamentale des formes expressives qui ramènent le sens vivant et philosophique des récits issus des cultures autochtones.

\section{La légende du windigo dans les récits littéraires et cinématographiques}

10 À l'encontre de la dépréciation, de l'aliénation et de l'enfermement que génèrent de telles constructions du savoir et des connaissances, des écrivains et des réalisateurs 
autochtones convoquent dans leurs créations des univers à la mesure de l'époque contemporaine. Par exemple, le long métrage Rimes pour revenants du réalisateur miqmaq Jeff Barnaby et le roman Le Mutilateur de l'écrivain atikamekw Julian Mahikan créent des mondes durs et inquiétants, légendaires et fantastiques, qui interrogent de façon créatrice différentes idées et réalités. D'autres œuvres recourent plus précisément à la légende du windigo. Pensons au roman Champion et Ooneemeetoo de l'écrivain cri Tomson Highway, au roman Le Chemin des âmes de l'écrivain d'origine anishnabe Joseph Boyden ou encore au long métrage $A$ Wintigo Tale du poète ojibwé Armand Garnet Ruffo. Du côté du cinéma autochtone émergent, plusieurs productions du Wapikoni mobile s'inspirent de récits autochtones se transmettant depuis de nombreuses générations. Le court métrage de fiction Windigo, réalisé en 2009 au lac Simon, dans la région de l'AbitibiTémiscamingue, par le cinéaste anishnabe Kris Happyjack-McKenzie ${ }^{6}$, en constitue un exemple éloquent. Ce film est particulièrement riche du point de vue narratif et esthétique. Il s'inscrit dans l'effervescence actuelle du cinéma autochtone au Québec, effervescence qui doit beaucoup à l'espace de création ouvert par le Wapikoni mobile, ce studio de cinéma ambulant à l'origine d'une production filmique autochtone créative, originale et d'une esthétique soignée, allant du documentaire au court métrage de fiction en passant par l'animation ${ }^{7}$. Le court métrage de Happyjack-Mckenzie met en scène la négociation d'une violence récurrente et effroyable, incarnée par un esprit affamé qui traque et engloutit les êtres ayant dévié de leur chemin. Cette violence prédatrice se laisse interpréter comme une violence coloniale se perpétuant, sans que cette thématique ne soit pourtant l'objet explicite de Windigo. En effet, si ce court métrage de fiction s'inscrit bien dans le contexte sociopolitique et culturel autochtone actuel auquel les images font référence, le film du réalisateur anishnabe - et c'est là toute sa force propose une réflexion philosophique élargie que la légende du windigo lui permet d'articuler.

11 Le film Windigo s'ouvre sur des images d'arbres d'une forêt obscurcie, en territoire anishnabe. Une narration en voix hors-champ, assurée par un aîné en langue anishnabe, raconte l'apparition, l'éloignement et le retour du windigo : « Il y a plusieurs siècles, la Nation Anishnabe du lac Nadaway rencontra une créature qui rôdait dans la nuit. Les anciens l'ont surnommée "Présence de la forêt". Cette présence emmenait les êtres égarés.» Ces premiers mots sont suivis d'un long plan fixe donnant à observer, au loin, une petite communauté autochtone à l'aspect déserté, tandis que la narration fait allusion à la force d'annihilation de cette présence qui «ne laissait aucune trace d'eux». La narration rappelle ensuite un moment déterminant, celui où « un homme courageux affronta la bête ». "Il réussit à blesser la créature, la mettant dans un long sommeil ", poursuit la voix de l'aîné au son du vent qui siffle, tandis qu'un long plan fixe donne à contempler une route forestière surplombée d'un ciel gris et nuageux. Le plan suivant nous amène à l'intérieur de la communauté, où l'aîné conclut son récit en disant : « On dit qu'aujourd'hui le sommeil de la bête fut interrompu, et qu'elle rôde à nouveau à la recherche d'une proie. » Dès que ces mots sont prononcés, le titre Windigo apparaît en lettres blanches au fond de l'écran noirci, la pleine lune en arrière-plan. Au son du tambour, une seconde narration en voix hors-champ, assurée cette fois par un jeune homme, prend en charge le récit : « Kiwetin et ses amis se promenaient dans le bois avec ses amis, là où la bête dormait. » Toujours en français, le narrateur constate le réveil de la bête, laquelle est attirée par l'odeur de la chair et du sang, "fascinée par les êtres humains qui ont peur ». Des images d'un écolier, adolescent, assis à son bureau en train 
de dessiner au crayon un visage maléfique, apparaissent à l'écran, signe que la nouvelle génération doit maintenant affronter cette créature. Happyjack-McKenzie met en scène la transmission d'un ancien récit anishnabe que le court métrage Windigo fait revivre en le racontant à son tour. Il contribue ainsi à rétablir des liens intergénérationnels mis à mal par les pratiques coloniales. En même temps, il rappelle que les êtres désorientés, isolés et craintifs constituent à toutes les époques des proies vulnérables aux esprits malveillants qui divisent pour mieux régner, à l'instar de celui qui, dans Windigo, « les a séparés, pour qu'il puisse les tuer un par un ».

On voit se dessiner dans Windigo une tension à négocier entre deux formes de continuité : celle d'une force destructrice, caractérisée par la répétition inlassable d'une violence prédatrice s'en prenant aux individus ayant perdu toute croyance, et celle d'une force protectrice rendue agissante grâce à une spiritualité retrouvée. Par un jeu de juxtaposition des images, le film met en parallèle, d'une part, une présence de la forêt faisant retour après un long sommeil et, d'autre part, l'intimidation infligée au protagoniste par des camarades de classe dans les couloirs d'une école de la communauté. Ces deux figurations de la violence prédatrice se fondent en une seule lorsque la présence de la forêt se manifeste dans les couloirs de l'établissement scolaire, et ce, sans pour autant cesser de rôder dans la forêt. Le double lieu d'agissements de la bête fait foi de sa mobilité. Il renvoie de plus à la négociation de deux modes de vie, l'un enraciné dans le territoire, l'autre encadré par les institutions. Le murmure rauque du windigo, le noir clignotement manifestant sa présence, le déplacement de la caméra dénotant sa progression entre les casiers de l'école, ainsi que la figure du désœuvrement et de l'aliénation incarnée par les adolescents rappellent la façon dont Tomson Highway et Joseph Boyden se servent de la figure du windigo pour « relier entre elles les institutions de la justice, de la police, de la prison, de la réserve, des pensionnats et de l'armée, suggérant que ces institutions sont responsables de l'état de siège imposé aux façons de vivre des Cris » (McCall 2013 : 64). Chez Happyjack-McKenzie, la résignation et l'effroi que l'on perçoit dans les yeux du protagoniste brutalisé par ses camarades de classe puis pourchassé par l'esprit du windigo dans les couloirs de l'école suggèrent de façon similaire un mal-être associé aux institutions coloniales.

Dans Windigo, c'est l'apparition d'un guide spirituel, représenté sous les traits d'une jeune femme autochtone d'une blancheur translucide, qui aidera le protagoniste à se réorienter et à affronter l'esprit malveillant qui le pourchasse. Également représentée par la riposte en langue anishnabe et le chant traditionnel l'accompagnant, la réhabilitation de la spiritualité et de la culture est particulièrement significative si l'on pense qu'elles ont été systématiquement la cible des politiques d'assimilation du gouvernement canadien. « Il a survécu et les autres ont péri ", indique la narration en voix off, évoquant l'expérience des Autochtones qui ont survécu et survivent toujours aux effets du traumatisme multigénérationnel et cumulatif, collectif et historique que décrivent les auteurs de l'article « Healing the American Indian Soul Wound » (E. Duran, B. Duran, Brave Heart et Horse-Davis 1998). De manière significative, la force de résister repose sur la foi, mais aussi sur la création de liens capables de contrer l'aliénation et l'affaiblissement affectant les êtres isolés. C'est ce qui se dessine dans la scène où la guide spirituelle se tient aux côtés du protagoniste, le soutenant dans son combat, de même que dans la séquence où un adolescent pourchassé rejoint le survivant avec lequel il prend la fuite. 


\section{Le windigo comme figuration d'une violence récurrente}

14 Si le protagoniste a survécu, le windigo, lui, demeure vivant et ne perd pas un instant pour se lancer à la poursuite des « esprits faibles ». Des images au ralenti donnent à voir deux adolescents en fuite sur une route forestière tandis que la narration avertit du danger sournois qui guette en chacun de nous :

Des fois, il va arriver que ta conscience te joue des tours, tu vas penser à des affaires dans ta tête... T'as jamais pensé à ça. Tu vas avoir des images noires que t'as jamais vues, tu vas rêver à des cauchemars que t'as jamais... Tu sais, t'as jamais rêvé à ces situations-là. Tu vas être initié à un mal.

Happyjack-Mckenzie évoque ici la possibilité toujours présente de se transformer en windigo, c'est-à-dire de devenir l'assaillant, le despote, le persécuteur, celui des autres, sans aucun doute, mais également le sien propre. Il pose la question de l'intériorisation de l'oppression menaçant tous ceux qui en subissent les affres, l'opprimé devenant à son tour l'oppresseur, et ainsi de suite dans une chaîne infinie, telle la faim insatiable du windigo. Dans une entrevue, le conteur anishinabe Louis Bird explique que s'il existe un windigo causé par la famine, il y a aussi «l'autre wihtiko, celui qui fut créé par le fait d'avoir été rendu complètement fou» (Bird 2002). C'est ce qui explique que des gens poussés à l'extrême de la déraison, que ce soit « par une force, par une autre personne ou par quelque chose d'autre qu'humain", ont pu finir par se transformer un jour en windigo.

Ailleurs dans le court métrage Windigo, la narration constate que la bête, ayant été contrecarrée par le protagoniste, part aussitôt à la chasse "d'autres proies qui ne croyaient en rien». Au son d'une musique inquiétante, on voit défiler des images ombragées du sol. Le spectateur entraîné dans l'action constate tout à coup qu'il est en train de marcher dans la forêt. Par un jeu d'identification avec la caméra subjective, il se retrouve dans la peau du windigo ; lui qui croyait regarder la scène à distance, l'observer d'un regard objectif, à la manière du spectateur ou de l'anthropologue, découvre qu'il a été happé, infecté, possédé, et que c'est lui qui est maintenant en train de traquer des jeunes de la communauté. Le film se conclut par un écran noirci, signe de l'omniprésence du windigo, puis les crédits défilent sur fond de marécage inquiétant, tandis que résonnent des cris de détresse et d'agonie provenant du fond des bois. Le dernier plan montre une chemise blanche suspendue à un arbre, signe que l'esprit du windigo a emporté à jamais une nouvelle victime. En forçant le spectateur à devenir le windigo au moyen d'un jeu d'identification avec la caméra subjective, le film fait allusion au potentiel de violence qui dort en chacun de nous. Il renvoie ainsi aux circonstances universelles par lesquelles, ainsi que l'explique Bird, une personne poussée à la limite de son humanité peut perdre la raison et devenir à son tour agent de l'oppression qu'elle a subie. Sans l'énoncer, le court métrage Windigo pose aussi la question de la responsabilité qui nous incombe face un héritage colonial dont nous puisons nos privilèges et dont les peuples autochtones continuent de se relever tant son poids demeure écrasant. En ce sens, il renvoie au caractère structurel du colonialisme qui continue de moduler les relations entre les peuples sur le continent américain. 


\section{La fiction cinématographique comme possible de la création contemporaine}

17 La fiction cinématographique constitue un volet porteur de la création autochtone contemporaine, notamment par les modes d'expression qu'elle permet d'explorer. La chercheure métis Kristina Fagan (2009) observe que le témoignage direct n'est pas une forme de communication privilégiée dans les cultures autochtones, lesquelles tendent plutôt à valoriser le discours indirect comme moyen de transmettre des connaissances, d'exposer les souffrances, d'exprimer sa colère et de critiquer sans engager de confrontation. De la même manière, le réalisateur anishnabe Kris Happyjack-Mckenzie propose une réflexion cinématographique sur la perte de sens, l'aliénation, l'intimidation et le caractère cyclique de la violence, et ce, sans expliciter clairement la nature de cette violence. Dans Windigo, Happyjack-McKenzie fait une critique des forces d'aliénation, mais en tendant vers l'harmonie et l'apaisement. Son propos rejoint encore une fois celui de l'écrivain anishnabe Basil H. Johnston, selon qui l'équilibre est garant de protection contre les affres de la cupidité humaine que représente le windigo (1995 : 223). Dans un contexte où les windigos, réduits à des superstitions vides de sens, « ont été arrachés du lieu qu'ils occupaient dans les traditions et la culture anishnabe » (Johnston 1995 : 235), la reterritorialisation de la légende du windigo que réalise par et dans la création Happyjack-McKenzie s'avère particulièrement significative. Fait notable, Windigo déconstruit une fiction médicale présentée comme un savoir objectif. Cette fiction, faut-il ajouter, a été créée par un corps médical qui a dépouillé une légende anishnabe de sa portée métaphorique et philosophique pour n'en garder que les os, c'est-à-dire en la cannibalisant presque entièrement pour n'en laisser qu'une forme de démence associée aux cultures autochtones. Le court métrage de Happyjack-McKenzie vient contrer les conceptions du primitivisme à l'origine de la construction médicale de la psychose du windigo, lesquelles tendent à opposer dégénérescence, dérèglement et pathogénèse, d'un côté, et, de l'autre, pureté, harmonie et vertus thérapeutiques (Waldram 2004 : 11). En articulant dans ce court métrage de fiction une réflexion philosophique inspirée de la cosmologie anishnabe, mais sans donner dans le cliché du noble sauvage à la Jean-Jacques Rousseau ou la médecine new age, le réalisateur anishinabe contribue à remédier aux effets néfastes de ce que Yves Sioui Durand décrit comme «la négation de la psyché profonde, des archétypes mythologiques qui enracinaient la culture» (2003: 60). De même, à l'encontre de «[l]'abandon forcé du nomadisme » (Ibid.), Windigo se rattache à l'histoire orale, laquelle est étroitement liée aux politiques de l'occupation du territoire tout autant qu'à une certaine conception de l'histoire et de la culture. Plutôt que de fournir un mode de conduite ou une clé d'interprétation, Happyjack-McKenzie nous fait don d'un récit cinématographique à partir duquel il nous invite à réfléchir aux dynamiques relationnelles au cœur de la condition humaine.

Le film Windigo rappelle ainsi l'idée avancée par des intellectuels autochtones tels Lee Maracle et Daniel Heath Justice, selon laquelle « la théorie de la décolonisation n'est pas restreinte aux essais critiques et aux analyses de texte, mais se trouve également enfouie dans le flot de signification de la poésie, du roman et de l'écriture de création " (Justice 2004 : 114). Il propose une réflexion philosophique qui se poursuit depuis des millénaires en pays autochtone $e^{8}$ une réflexion sur les monstres qui nous habitent, sur le vide et sur le croire, sur les différents modes d'appréhension du monde, jadis comme aujourd'hui, dans 
notre monde globalisé en proie à une violence systémique. Ce faisant, il redéploie tout le potentiel éducatif et heuristique de la légende du windigo ; il l'actualise et, par le biais du médium cinématographique, le fait circuler dans les communautés autochtones dans le même temps qu'il ouvre à une diffusion élargie. Lyons fait observer que la souveraineté, loin de représenter une enclave, constitue plutôt « la capacité de s'affirmer, renouvelé, en présence des autres » (2000: 457). C'est précisément en ce sens que le court métrage de Happyjack-McKenzie, en reprenant une légende de la tradition orale anishinabe pour faire sens des réalités contemporaines, agit à la fois comme acte souverain et moyen de transmission des connaissances.

\section{BIBLIOGRAPHIE}

Bird, Louis, 2002, « Our Voices-Mystery Stories $1 »$, Recorded on January 31, transcrit par Youngok Kang-Bohr, [En ligne], http://www.ourvoices.ca/filestore/pdf/0/1/3/0/0130.pdf, consulté le 9 décembre 2015.

-, 2013, "Wihtigo, Or the Consequences of Not Listening ", dans D. David Moses, T. Goldie et A. Ruffo (dir.), Anthology of Canadian Native Literature in English, $4^{\mathrm{e}}$ éd., Don Mills, Oxford UP, p. 126-129.

DePasquale, Paul, Eigenbrod, Renate et LaRocque, Emma (dir.), 2009, Across Cultures. Across Borders Canadian Aboriginal and Native American Literatures, Peterborough, Broadview Press.

Duran, Eduardo, Duran, Bonnie, Brave Heart, Maria Yellow Horse et Horse-Davis, Susan Yellow, 1998, « Healing the American Indian Soul Wound », dans Y. Danieli (dir.), International Handbook of Multigenerational Legacies of Trauma, New York, Plenum Press, p. 341-354.

Fagan, Kristina, 2009, « Weesageechak Meets the Weetigo: Storytelling, Humour, and Trauma in the Fiction of Richard, Tomson Highway, and Eden Robinson ", Études en littérature canadienne, vol. $34, \mathrm{n}^{\circ} 1$, p. 204-226.

Gatti, Maurizio (dir.), 2008, Mots de neige, de sable et d'océan. Littératures autochtones. Québec, Maroc, Polynésie française, Nouvelle-Calédonie, Algérie, préf. de Tomson Highway, Wendake, Les Éditions du CDFM.

Johnston, Basil H., 1995, The Manitous: The Spiritual World of the Ojibway, New York, Harper Collins. Justice, Daniel Heath, 2004, « Seeing (and Reading) Red. Indian Outlaws in the Ivory Tower », dans D. A. Mihesuah et A. C. Wilson (dir.), Indigeneizing the Academy. Transforming Scholarship and Empowering Communities, Lincoln, University of Nebraska Press, p. 110-123.

Lainey, Jonathan, 2013, « Les colliers de wampum comme support mémoriel : le cas du Two-Dog Wampum », dans A. Beaulieu, S. Gervais et M. Papillon (dir.), Les Autochtones et le Québec. Des premiers contacts au Plan Nord, Montréal, Les Presses de l'Université de Montréal, p. 93-111.

Lyons, Scott Richard, 2000, «Rhetorical Sovereignty: What Do American Indians Want from Writing? », College Composition and Communication, vol. 51, $\mathrm{n}^{\circ}$ 3, p. 447-468. 
McCall, Sophie, 2013, «Intimate Enemies. Weetigo, Weesageechak, and the Politics of Reconciliation in Tomson Highway's Kiss of the Fur Queen and Joseph Boyden's Three Day Road », SAIL, vol. 25, $\mathrm{n}^{\circ}$ 3, p. 57-85.

Paré, François, [1992] 2001, Les Littératures de l'exiguïté, préf. de Robert Major, Ottawa, Le Nordir, coll. «BCF ».

Sioui Durand, Guy, 2007, « Maurizio GATTI, Être écrivain amérindien au Québec. Indianité et création littéraire ", Recherches sociographiques, vol. 48, n 2, p. 183-186.

- (dir.), 2010, Inter, art actuel, $\mathrm{n}^{\circ}$ 104, dossier « Indiens Indians Indios ».

Sioui Durand, Yves, 2003, « Kaion'ni, le wampum rompu. De la rupture de la chaîne d'alliance ou "le grand inconscient résineux" ", Recherches amérindiennes au Québec, vol. 33, n 3, p. 55-64.

-, 2009, «Y a-t-il un nouveau monde pour les Amérindiens ? », dans N. Gagné, M. Thibault et M. Salaün (dir.), Autochtonies. Vues de France et du Québec, Québec, Les Presses de l'Université Laval et DIALOG, p. 505-514.

St-Amand, Isabelle, 2010, « Discours critiques pour l'étude de la littérature autochtone dans l'espace francophone du Québec ", Études en littérature canadienne, vol. 35, nº 2, p. 30-52.

Waldram, James B., 2004, Revenge of the Windigo: The Construction of the Mind and Mental Health of North American Aboriginal Peoples, Toronto, University of Toronto Press.

Womack, Craig S., Justice, Daniel Heath et Teuton, Christopher B., 2008, Reasoning Together. The Native Critics Collective, Norman, University of Oklahoma Press.

\section{NOTES}

1. Traduction libre de la citation originale : « Finally it got dark. Supper had already been served. Everybody had eaten for the last time, and the elders were only sitting around. And the young boy was still talking about the wihtigo that never showed up. But all of a sudden there was a terrible feeling of fear. The young boy, especially, was just gripped by fear. He started to tell his mother that he was scared, but his grandmother says, "Don't you say anything! You have asked for it!" And sure enough, a wihtigo was near. And, as usually happened when a wihtigo was close by, they started to hear the sound of a heartbeat-that was the usual indication of the nearness of a wihtigo amongst our ancestors. The old lady recognized the sound and says, "See what I say? See what I told you? It never fails." "

2. Le wampum, collier de perles fabriqué de coquillages marins, est une archive de la tradition orale autochtone. En plus de servir de support mémoriel, les colliers de wampum sont « offerts lors des rencontres formelles pour appuyer le discours prononcé, pour le rendre légitime et officiel » (Lainey 2013 : 96-97).

3. Nous traduisons librement toutes les citations de l'article.

4. « Dès les années 1870, le gouvernement du Canada a commencé à financer un système de pensionnats indiens administrés chacun par une congrégation religieuse. L'un des objectifs consistait à soustraire les enfants autochtones à l'influence de leurs parents et de leur culture pour les intégrer à la société générale. Les enfants étaient séparés de leur famille et de leur communauté et on leur interdisait de parler leur langue. Le dernier pensionnat a fermé ses portes dans les années 1990. Selon les estimations, quelque 150000 enfants inuits, métis ou des Premières nations sont passés par ces pensionnats. " (Bureau du vérificateur général du Canada, http://www.oag-bvg.gc.ca/internet/Francais/parl_oag_201304_06_f_38191.html\#hd5a, consulté le 8 mai 2015.) 
5. http://www.pm.gc.ca/fra/nouvelles/2008/06/11/premier-ministre-harper-presente-desexcuses-completes-au-nom-des-canadiens\#sthash.8wwGVGwF.dpuf (consulté le 10 janvier 2014). 6. Le film est en ligne : http://www.wapikoni.ca/films/windigo-2009 (consulté le 10 janvier 2014). 7. Le Wapikoni mobile a été cofondé en 2003 par Manon Barbeau, le Conseil de la Nation Atikamekw et le Conseil des jeunes des Premières Nations du Québec et du Labrador, avec le soutien de l'Assemblée des Premières Nations et la collaboration de l'Office national du film du Canada. Depuis 2004, le Wapikoni mobile circule dans les communautés autochtones et offre aux jeunes des Premières Nations des ateliers permettant la maîtrise des outils numériques par la réalisation de courts métrages et d'œuvres musicales. À chacune de ses escales, des « cinéastesaccompagnateurs" accueillent une trentaine de jeunes participants en formation à toutes les étapes de la réalisation. À ce jour, plus de 3000 jeunes ont été formés ou initiés au cinéma documentaire ou à l'enregistrement musical. Le Wapikoni mobile a donné lieu à la création d'une collection unique au monde de près de plus de 750 films et 450 musiques, une contribution exceptionnelle au patrimoine culturel des Premières Nations. Le projet élargit actuellement son réseau de co-création audiovisuelle autochtone à l'échelle continentale, notamment par l'entremise du Réseau international de création audiovisuelle autochtone (RICAA). Source: Wapikoni mobile, http://www.wapikoni.ca/a-propos/quest-ce-que-le-wapikoni/historique (consulté le 12 mai 2015).

8. À ce sujet, voir entre autres Womack, Justice et Teuton (2008).

\section{RÉSUMÉS}

À partir des notions d'éthique et de souveraineté élaborées dans le domaine des études littéraires autochtones en Amérique du Nord, cet article propose une réflexion sur le recours à la légende $\mathrm{du}$ windigo dans la création autochtone contemporaine, plus particulièrement en contexte francophone. Par contraste avec la construction occidentale de la psychose du windigo, l'article met en avant la conceptualisation d'une violence récurrente articulée dans le court métrage de fiction Windigo du réalisateur anishnabe Kris Happyjack-McKenzie.

Working with the notions of ethics and sovereignty developed in the field of Indigenous literary studies in North America, this article examines the uses of the legend of the windigo in contemporary Indigenous creation, more specifically in a francophone context. In contrast with the Western construction of the windigo psychosis, this article focuses on the conceptualization of a recurring violence in the short fiction film Windigo by Anishnabe director Kris HappyjackMcKenzie.

\section{INDEX}

Mots-clés : littérature autochtone, cinéma autochtone, approches théoriques, Wapikoni mobile, Québec

Keywords : Indigenous literature, Indigenous cinema, critical approaches, Wapikoni mobile, Quebec 


\section{AUTEUR}

\section{ISABELLE ST-AMAND}

Université du Manitoba 